\title{
Qualité Des Eaux De Boisson Conditionnées En Sachet Vendues Dans La Région De Dakar Au Sénégal
}

\author{
Modou Dieng \\ Laboratoire d'analyses et Essais, Ecole Supérieure Polytechnique, Université \\ Cheikh Anta Diop (UCAD), Dakar, Sénégal \\ Janvier Kindossi \\ Département de Nutrition et Sciences Agroalimentaires, Faculté \\ d’Agronomie, Université de Parakou, Parakou, Bénin \\ Noumbé Diop \\ Ecole Supérieure Polytechnique, Département Génie chimique et Biologie \\ appliquée, UCAD, Dakar, Sénégal

\section{Mbaye Mbengue} \\ Laboratoires de Bactériologie et Pathologie Aviaire (LBPA), ISRA, Dakar, \\ Sénégal
}

Doi:10.19044/esj.2021.v17n21p104

Submitted: 27 August 2020

Accepted: 27 April 2021

Published: 30 June 2021
Copyright 2021 Author(s)

Under Creative Commons BY-NC-ND 4.0 OPEN ACCESS

Cite As:

Dieng M., Kindossi J., Diop N. \& Mbengue M. (2021). Qualité Des Eaux De Boisson Conditionnées En Sachet Vendues Dans La Région De Dakar Au Sénégal. European Scientific Journal, ESJ, 17(21), 104.

https://doi.org/10.19044/esj.2021.v17n21p104

\section{Résumé}

La qualité de l'eau de boisson conditionnée en sachet, vendue sous différentes marques dans les marchés de Dakar a été étudiée. Un total de 60 échantillons d'eau conditionnée dans des sachets sous 15 différentes marques a été prélevé de façon aléatoire dans les 5 différents marchés de Dakar pour déterminer la qualité physicochimique et microbiologique. Les résultats ont montré que la qualité physicochimique des eaux conditionnées en sachet est conforme à la norme réglementaire Norme NF EN ISO 19458 en la matière. Cependant, en ce qui concerne la qualité microbiologique, $83 \%$ des échantillons ont été contaminés avec une forte charge de la flore mésophile aérobie totale supérieure à celle de la norme NF EN ISO 19458 et 15\% des échantillons sont vigoureusement contaminés par les coliformes totaux. 
Cependant, aucune présence de salmonelle n'est enregistrée dans les échantillons étudiés. Malgré tout, la population de Dakar qui utilise cet eau s'expose à des risques sanitaires. Il convient au service d'hygiène de sensibiliser et de contrôler l'activité de la vente de l'eau conditionnée en sachet.

Mots clés: Eau En Sachet, Qualité, Consommation, Contamination, Dakar Sénegal

\title{
Quality Of Packaged Beverage Waters Sold In Sénégal, Dakar Region
}

\section{Modou Dieng}

Laboratoire d'analyses et Essais, Ecole Supérieure Polytechnique, Université Cheikh Anta Diop (UCAD), Dakar, Sénégal

\section{Janvier Kindossi}

Département de Nutrition et Sciences Agroalimentaires, Faculté d’Agronomie, Université de Parakou, Parakou, Bénin

\section{Noumbé Diop}

Ecole Supérieure Polytechnique, Département Génie chimique et Biologie appliquée, UCAD, Dakar, Sénégal

\section{Mbaye Mbengue}

Laboratoires de Bactériologie et Pathologie Aviaire (LBPA), ISRA, Dakar, Sénégal

\begin{abstract}
The quality of drinking water packaged in plastic bags through different brands sold in Dakar markets has been studied. 60 samples packaged through 15 different brands were taken randomly from the 5 different markets to determine their physico-chemical and microbiological quality. The results showed that physicochemical quality complies with the standards in this area. However, with regard to microbiological quality, $83 \%$ of the samples were contaminated at a high load with total viable counts, higher than the standard and $15 \%$ of the samples are highly contaminated with total coliforms. Meanwhile, there is no salmonella contamination was recorded in the samples studied. As a result, the population of Dakar who relies to this drinking water supply is exposed to health risks. The hygiene service should raise awareness and monitor the activity of selling drinking water.
\end{abstract}

Keywords: Sachet Water, Quality, Consumption, Contamination, Dakar Senegal 


\section{Introduction}

Dans le monde, plus particulièrement en Afrique, le risque sanitaire le plus enregistré est lié à la consommation de l'eau souillée. Ce fait constitue une véritable problématique en matière de santé publique (Benajiba et al. 2013; Santsa Nguefack et al. 2018). Pour pallier cette difficulté d'accès à l'eau de boisson de bonne qualité dans les pays en développement, et notamment en Afrique subsaharienne, plusieurs rencontres scientifiques sur l'eau et l'assainissement ont eu lieu à la Haye en 2000, à Kyoto en 2003, à Mexico en 2006 et au Sénégal en 2012 (ANSD, 2015). Les retombées de ces rencontres ont permis l'installation de l'eau du robinet, une eau de boisson saine de qualité garantissant une meilleure santé des populations. Malgré, une accessibilité de plus en plus importante de l'eau de robinet au Sénégal, la population de Dakar semble préférer de plus en plus l'eau conditionnée en sachet et conservé au frais, du fait de son caractère rafraichissant et de son coût abordable. Pour profiter de cette opportunité, beaucoup d’entrepreneurs privés se sont lancés dans la distribution d'eau de boisson conditionnée en sachet sous différentes marques.

De nos jours, il a été constaté une prolifération de marques d'eaux en sachet fabriquées localement et vendues dans les marchés. Ces sachets d'eau répondent non seulement à un besoin Ba Eau Bab Sénégal pratique pour les consommateurs, mais constituent une source de revenus pour les commerçants.

Ainsi, sur la journée, des milliers de sachets d'eau sont vendus. Les eaux de boisson dont la qualité n'est pas garantie envahissent la ville de Dakar. Leur provenance et les conditions de production échappent souvent aux autorités en charge de la salubrité des aliments. Selon L’OMS (2017), la consommation d'une eau insalubre expose les populations à plusieurs risques notamment les maladies d'origines hydriques. Diverses études ont mis en évidence la pollution des eaux par les microorganismes. Les études réalisées par Benajiba et al. (2013), Soncy et al. (2015), Hounsinou et al. (2015) et Zerhouni et al. (2015) ont révélé une importante présence de flore mésophile aérobie totale et une contamination fécale due à la présence de Coliformes totaux, de Coliformes thermotolérants et d'Entérocoques.

L’objectif de ce travail est d'étudier la qualité physicochimique et microbiologique des eaux conditionnées en sachets afin de mettre en évidence les éventuels risques de maladies à transmission hydrique auxquelles sont exposées les populations sénégalaises en général et dakaroise en particulier consommatrices de ces types d'eaux. 


\section{Matériel et Méthodes Zone d'étude}

La région de Dakar d'une superficie de $550 \mathrm{~km}^{2}$ soit $0,28 \%$ de la superficie nationale, est située dans la presqu'île du Cap Vert. Elle est comprise entre les coordonnées $17^{\circ} 10$ et $17^{\circ} 32$ de longitude Ouest et les coordonnées $14^{\circ} 53$ et $14^{\circ} 35$ de latitude Nord. Elle est limitée à l'Est par la région de Thiès et par l'Océan Atlantique dans ses parties Nord, Ouest et Sud (Figure 1). La région de Dakar a été choisie compte tenu de sa position de ville carrefour de grosses unités industrielles assurant la transformation des produits locaux destinés à l'exportation, la manufacture de produits importés destinés au marché local national, de sa dense population et pour son caractère cosmopolite.

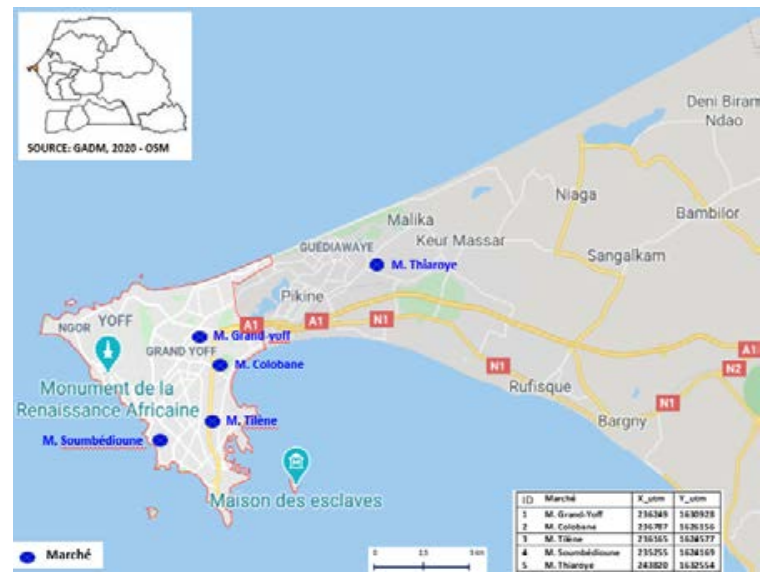

Figure 1. Lieux de prélèvements

\section{Échantillonnage}

D’août à septembre 2018, un échantillonnage d'eau conditionnée dans des sachets plastiques a été effectué dans les marchés de Tilène, Thiaroye, Colobane, Grand-Yoff et Soumbédioune qui sont les cinq (05) grands marchés de la région de Dakar (Figure 1). Soixante (60) échantillons d'eau conditionnée dans des sachets de 300 à $500 \mathrm{ml}$ ont été prélevés de façon aléatoire dans les 5 marchés de Dakar choisis. Quinze (15) marques de sachets d'eau ont été identifiées et prélevées à raison de 4 échantillons par marque. Les résultats auront pour objectif de sensibiliser les promoteurs et la population. Pour cela, les marques sont numéros de 1 à 15. Les échantillons prélevés ont été prélevés et étiquetés soigneusement puis déposés dans une glacière munie de carboglaces avant d'être acheminés au laboratoire (ISO 5667-3, 2004 ; ISO 5667-1, 2007). Les étiquettes portent un numéro, le nom de la marque et la date de prélèvement. Les analyses microbiologiques ont été effectuées au niveau du Laboratoire National de l’Elevage et des Recherches 
Vétérinaires plus particulièrement aux Laboratoires de Bactériologie et Pathologie Aviaire (LBPA).

\section{Analyse chimique et bactériologique de l'eau conditionnée en sachet}

Le $\mathrm{pH}$ a été mesuré sur le terrain à l'aide d'un multi-paramètre portatif de marque HANNA model HI 9829 de précision 0,01. La turbidité (exprimées en NTU) a été mesurée grâce à un turbidimètre portable de marque OrbicoHillige modèle 966 (ISO 7027, 2007). La dureté totale est déterminée par titrimétrie à l'EDTA (NF T90-003, 1984).

En ce qui concerne la qualité microbiologique, les analyses généralement effectuées sur une eau de consommation humaine sont basées sur l'identification et le dénombrement des germes dans les échantillons à analyser (ISO 5667-3, 2004 ; ISO 19458, 2006). Il s'agit donc :

- d'une part, pour l'analyse qualitative, de la recherche d'un indicateur de contamination fécale, Salmonella, suivant la méthode ISO 6579 (2002). La recherche de Salmonella consiste en la détermination de la présence ou de l'absence du genre Salmonella suite à une étape de préenrichissement, d'enrichissement, d'isolement et d'identification à une température d'incubation de $37^{\circ} \mathrm{C}$ pendant 24 heures. Le Préenrichissement est réalisé sur milieu eau peptonnée tamponnée, L’Enrichissement sur milieu bouillon au sélénite de sodium, l'Isolement sur gélose Salmonella-Shigella (gélose SS) et sur gélose Hektoen. Enfin, l'identification a été réalisée sur galerie API $20^{\mathrm{E}}$.

- Et d'autre part, pour les analyses quantitatives, d'un dénombrement de la flore mésophile aérobie totale (FMAT) en milieu solide PCA (Plate Count Agar) selon la méthode ISO-4833 (2003) et d'un dénombrement des coliformes totaux (CT) sur la gélose lactosée biliée au vert brillant (VRBL) selon la méthode AFNOR V 08-060.

\section{Analyse statistique}

Le traitement de l'ensemble de données a été réalisé avec le Tableur Excel 2010. Ces résultats d'analyses chimique et bactériologique ont été comparés aux Valeurs Guides OMS de la qualité de l'eau destinée à la consommation humaine. Ils ont été soumis à une analyse de variance (ANOVA) à un facteur suivi du test de Newman-keuls grâce au logiciel Statistica version 7.1 StatSoft France (2006). La signification statistique de la différence entre les échantillons d'eau conditionnée par numéro de marque a été déterminée au seuil de $5 \%$.

\section{Résultats}

Caractéristiques physicochimiques des eaux de boissons conditionnées en sachet sous différentes marques 
Le Tableau 1 présente les caractéristiques physicochimiques des eaux de boisson conditionnées en sachet sous différentes marques. Les eaux de boisson conditionnées en sachet ont un $\mathrm{pH}$ variant de 7,12 à 7,36. Il n'y a pas de différence significative $(\mathrm{p}>0,05)$ entre les différentes valeurs de $\mathrm{pH}$ déterminées. Ces $\mathrm{pH}$ sont conformes aux Valeurs Guides recommandées par l'OMS qui sont comprises entre 6,5 et 9,5.

La turbidité des eaux varie de 0,10 à 0,61 NTU. Les valeurs de la turbidité des eaux de boisson conditionnées dans les sachets des marques 2, 3 , 4 , et 15 sont significativement supérieures au seuil de $5 \%$ à celles des eaux de boisson conditionnées dans les sachets des autres marques collectées. Cela montre que ces eaux de boisson contiennent plus de particules en suspensions que les autres. Toutefois, tous les échantillons présentent des valeurs très inférieures à celles recommandée par les Valeurs Guides OMS (inférieure à 5 NTU).

La dureté des eaux varie de 24,50 à $34 \mathrm{mg} / \mathrm{ml}$ de $\mathrm{CaCO}_{3}$. Ces valeurs de la dureté des eaux sont très faibles par rapport aux Valeurs Guides OMS qui sont comprise entre 150 et $500 \mathrm{mg} / \mathrm{ml}$ de $\mathrm{CaCO}_{3}$.

Tableau 1. Caractéristiques physico-chimiques des eaux de boissons conditionnées en sachet sous différentes marques

\begin{tabular}{|c|c|c|c|c|}
\hline $\mathrm{N}^{\circ}$ marque & $\begin{array}{l}\text { Nombre } \\
\text { d'échantillons } \\
\text { analysés }\end{array}$ & pH & $\begin{array}{l}\text { Turbidité } \\
\text { (NTU) }\end{array}$ & $\begin{array}{l}\begin{array}{l}\text { Dureté } \\
(\mathrm{mg} / \mathrm{l}\end{array} \\
\left.\mathrm{CaCO}_{3}\right)\end{array}$ \\
\hline Marque 1. & 4 & $7,18 \pm 0,08^{\mathrm{a}}$ & $0,16 \pm 0,03^{\mathrm{a}}$ & $32,00 \pm 0,00^{\mathrm{a}}$ \\
\hline Marque 2. & 4 & $7,36 \pm 0,11^{\mathrm{a}}$ & $0,45 \pm 0,05^{b}$ & $32,00 \pm 0,01^{\mathrm{a}}$ \\
\hline Marque 3. & 4 & $7,27 \pm 0,05^{\mathrm{a}}$ & $0,51 \pm 0,18^{\mathrm{b}}$ & $34,00 \pm 0,02^{\mathrm{a}}$ \\
\hline Marque 4. & 4 & $7,12 \pm 0,18^{\mathrm{a}}$ & $0,60 \pm 0,05^{b}$ & $33,50 \pm 2,50^{\mathrm{ab}}$ \\
\hline Marque 5. & 4 & $7,21 \pm 0,09^{\mathrm{a}}$ & $0,31 \pm 0,02^{\mathrm{a}}$ & $30,50 \pm 0,50^{\text {bc }}$ \\
\hline Marque 6. & 4 & $7,24 \pm 0,02^{\mathrm{a}}$ & $0,20 \pm 0,04^{\mathrm{a}}$ & $28,00 \pm 0,00^{\text {cd }}$ \\
\hline Marque 7. & 4 & $7,35 \pm 0,03^{\mathrm{a}}$ & $0,22 \pm 0,04^{\mathrm{a}}$ & $28,00 \pm 0,00^{\text {cd }}$ \\
\hline Marque 8. & 4 & $7,21 \pm 0,02^{\mathrm{a}}$ & $0,38 \pm 0,04^{\mathrm{a}}$ & $28,00 \pm 0,00^{\text {cd }}$ \\
\hline Marque 9. & 4 & $7,26 \pm 0,02^{\mathrm{a}}$ & $0,19 \pm 0,08^{\mathrm{a}}$ & $26,50 \pm 0,29^{\text {de }}$ \\
\hline Marque 10. & 4 & $7,23 \pm 0,02^{\mathrm{a}}$ & $0,22 \pm 0,02^{\mathrm{a}}$ & $26,00 \pm 0,00^{\mathrm{de}}$ \\
\hline Marque 11. & 4 & $7,26 \pm 0,02^{\mathrm{a}}$ & $0,10 \pm 0,02^{\mathrm{a}}$ & $26,00 \pm 0,00^{\text {de }}$ \\
\hline Marque 12. & 4 & $7,23 \pm 0,01^{\mathrm{a}}$ & $0,14 \pm 0,02^{\mathrm{a}}$ & $26,00 \pm 0,01^{\mathrm{de}}$ \\
\hline Marque 13. & 4 & $7,22 \pm 0,01^{\mathrm{a}}$ & $0,23 \pm 0,06^{\mathrm{a}}$ & $24,00 \pm 0,71^{\mathrm{e}}$ \\
\hline Marque 14. & 4 & $7,26 \pm 0,05^{\mathrm{a}}$ & $0,11 \pm 0,02^{\mathrm{a}}$ & $24,00 \pm 0,00^{\mathrm{e}}$ \\
\hline Marque 15. & 4 & $7,36 \pm 0,01^{\mathrm{a}}$ & $0,61 \pm 0,15^{\mathrm{b}}$ & $24,50 \pm 0,50^{\mathrm{e}}$ \\
\hline $\begin{array}{l}\text { Valeur } \\
\text { Maximale } \\
\text { admise (OMS) }\end{array}$ & & $6,5<\mathrm{pH}<9,5$ & Turbidité $<5$ & $\begin{array}{l}150<\text { Dureté < } \\
500\end{array}$ \\
\hline
\end{tabular}

Caractéristiques microbiologiques des eaux de boissons conditionnées en sachet sous différentes marques

Les caractéristiques microbiologiques des eaux de boisson conditionnées en sachet sous différentes marques sont présentées dans le 
Tableau 2. La flore mésophiles aérobie totale (FMAT) varie de 2,06 à 4,78 $\log _{10} \mathrm{UFC} / \mathrm{ml}$ d'eau de boisson. Aucune différence significative $(\mathrm{p}>0,05)$ n'est observée pour la FMAT des eaux de boisson. Ces valeurs de la FMAT enregistrées sont très supérieures à celles des recommandations de l'OMS qui sont inférieures ou égales à $1,30 \log _{10} \mathrm{UFC} / \mathrm{ml}$ d'eau de boisson.

Les coliformes totaux considérés comme des indicateurs de pollution varient de $<1$ à $1,37 \log _{10} \mathrm{UFC} / 100 \mathrm{ml}$. Aucune différence significative ( $\mathrm{p}>$ $0,05)$ n'est observée pour les coliformes totaux des eaux de boisson.

Les bactéries pathogènes comme les salmonelles n’ont été détectées.

Tableau 2. Caractéristiques microbiologiques des eaux de boissons conditionnées en sachet sous différentes marque

\begin{tabular}{|l|l|l|l|l|}
\hline $\mathbf{N}^{\circ}$ marque & $\begin{array}{l}\text { Nombre } \\
\text { d'échantillons } \\
\text { analysés }\end{array}$ & $\begin{array}{l}\mathbf{F A M T} \\
\mathbf{3 7}^{\circ} \mathbf{C} \\
\mathbf{L o g}_{\mathbf{1 0}} \\
\mathbf{( U F C / m l )}\end{array}$ & $\begin{array}{l}\text { Coliformes totaux à } \\
\mathbf{3 7}^{\circ} \mathbf{C} \\
\mathbf{L o g}_{\mathbf{1 0}} \text { (UFC/100ml) }\end{array}$ & $\begin{array}{l}\text { Salmonelles } \\
\mathbf{L o g}_{\mathbf{1 0}} \\
\text { (UFC/250ml) }\end{array}$ \\
\hline Marque 1. & 4 & $2,96 \pm 1,23$ & $1,37 \pm 0,36$ & Absence \\
\hline Marque 2. & 4 & $3,34 \pm 0,82$ & $<1$ & Absence \\
\hline Marque 3. & 4 & $3,24 \pm 0,73$ & $<1$ & Absence \\
\hline Marque 4. & 4 & $2,46 \pm 0,98$ & $<1$ & Absence \\
\hline Marque 5. & 4 & $4,11 \pm 0,87$ & $1,36 \pm 0,79$ & Absence \\
\hline Marque 6. & 4 & $3,77 \pm 0,85$ & $<1$ & Absence \\
\hline Marque 7. & 4 & $4,11 \pm 0,81$ & $<1$ & Absence \\
\hline Marque 8. & 4 & $2,09 \pm 1,39$ & $1,30 \pm 0,74$ & Absence \\
\hline Marque 9. & 4 & $2,51 \pm 1,32$ & $<1$ & Absence \\
\hline Marque 10. & 4 & $4,29 \pm 1,21$ & $<1$ & Absence \\
\hline Marque 11. & 4 & $3,87 \pm 0,94$ & $<1$ & Absence \\
\hline Marque 12. & 4 & $2,81 \pm 1,15$ & $<1$ & Absence \\
\hline Marque 13. & 4 & $3,12 \pm 1,40$ & $<1$ & Absence \\
\hline Marque 14. & 4 & $2,06 \pm 0,69$ & $<1$ & Absence \\
\hline Marque 15. & 4 & $4,78 \pm 0,71$ & $<1$ & Absence \\
\hline $\begin{array}{l}\text { Valeur } \\
\text { Maximale } \\
\text { admise } \\
\text { (OMS) }\end{array}$ & & $\leq 1,30$ & $<1$ & Absence \\
\hline
\end{tabular}

\section{Discussion}

L'eau est un élément indispensable pour la vie de l'homme et de celle des autres êtres vivants ; sa présence en quantité et qualité concourt au maintien de la santé (OMS, (2017). L’eau de boisson conditionnée dans des sachets constitue donc un aliment le plus consommé surtout dans les villes et les marchés subsahariens où elle est fréquemment utilisée pour la désaltération lors des périodes de grandes chaleurs.

L'étude des caractéristiques physicochimiques des eaux de boisson conditionnées en sachet suivant différentes marques vendues dans les marchés 
de Dakar a montré que ces eaux ont une qualité physicochimique conforme aux recommandations de l'OMS pour l'eau de boisson.

Toutefois, sur le plan microbiologique ces eaux de boisson sont contaminées par la flore mésophile aérobie totale (FMAT) et les germes coliformes totaux.

En fait, le dénombrement de la flore mésophile aérobie totale vise à estimer la densité de la population bactérienne générale présente dans l'eau de boisson, la plupart de ces germes constituant cette flore ne sont pas pathogènes. Cependant, certaines espèces peuvent être pathogènes opportunistes et causent des infections chez les personnes dont le système immunitaire est affaibli. La FAMT présente peu d'intérêt pour déceler la pollution d'origine fécale mais elle permet d'estimer l'état de salubrité de l'eau.

Le Tableau 3 montre que 83\% des eaux de boisson conditionnées en sachet de différentes marques contiennent une flore mésophile aérobie totale supérieure à la norme. Ces échantillons contaminés sont non satisfaisants contre à peine $17 \%$ qui sont satisfaisants. De ce fait, la forte concentration en germes totaux génère des problèmes d'ordre organoleptique de l'eau. Cette flore constitue donc un bon indicateur pour ce qui est de l'application des bonnes pratiques d'hygiène.

Rodier et al. (2013) ont évoqué que la qualité microbiologique des eaux de boisson est habituellement la principale responsable des cas de maladies infectieuses provoquées par les bactéries, virus, protozoaires et helminthes pathogènes. Ces microorganismes sont responsables des risques sanitaires associés aux eaux de boisson les plus répandues.

Dans ce cas, la consommation de ces eaux polluées n'est pas sans conséquence sur la santé de la population de Dakar qui consomme ces eaux. Notons que Kouadio et al. (1998) ont fait la même observation dans les communes d'Abidjan où les eaux en sachet vendues aux abords des écoles ont été révélées de mauvaise qualité microbiologique et chimique. Ils ont mentionné que les consommateurs s'exposent à des risques sanitaires.

Le Tableau 4 présente le taux de contamination des eaux de boissons par les coliformes totaux. En fait, 15\% des échantillons sont contaminés par les coliformes totaux. Les coliformes totaux ne sont pas des indications de pollution fécale seulement, leur origine peut être aussi environnementale (sol, végétation, eau) (Rodier, 2009). Ces bactéries coliformes sont en majorité du genre Escherichia, Citrobater, Klebsiella et Enterobacter, etc... (Rodier, 2009).

Ces bactéries ne sont généralement pas dangereuses du point de vue sanitaire sauf en cas de prolifération abondante ou de sensibilité particulière du consommateur. Toutefois, Rodier et al. (2009) ont souligné que leur 
présence en grand nombre signifie qu'ils peuvent véhiculer des microorganismes pathogènes.

La présence des coliformes totaux dans certains échantillons témoigne d'une hygiène défectueuse dans la transformation.

En ce qui concerne les Salmonelles l'analyse témoigne de leur absence dans tous les échantillons. De ce fait, ces eaux conditionnées en sachets ne transmettent pas la salmonellose qui augmente le risque d'une issue fatale surtout chez les personnes âgées (OMS, 2017).

Tableau 3. Niveau de contamination des eaux de boisson par la flore mésophile aérobie totale

\begin{tabular}{|l|l|l|}
\hline & Nombre d'échantillons & Pourcentages \\
\hline Contaminés & 50 & $83 \%$ \\
\hline Non contaminés & 10 & $17 \%$ \\
\hline
\end{tabular}

Tableau 4. Niveau de contamination des eaux de boisson par les coliformes totaux

\begin{tabular}{|l|l|l|}
\hline & Nombre d'échantillons & Pourcentages \\
\hline Contaminés & 9 & $15 \%$ \\
\hline non contaminés & 51 & $75 \%$ \\
\hline
\end{tabular}

\section{Conclusion}

Cette étude a montré que les eaux conditionnées en sachet sous différentes marques vendues dans les marchés de Dakar ont une qualité physicochimique conforme aux recommandations mais la qualité microbiologique est inadéquate avec une majorité (83\%) des eaux de boisson contaminée par des germes bactériologiques. La présence de ces germes bactériologiques implique une vigilance accrue car ils indiquent un risque pour la santé de la population de Dakar qui consomme ces eaux. Il est donc nécessaire de prévenir les consommateurs du risque que représentent ces eaux pour la santé. De ce fait, les services d'hygiène sont appelés à une sensibilisation, et au contrôle de l'activité de la vente de l'eau de boisson conditionnée en sachet afin de prévenir la population des cas de maladies hydriques.

\section{Remerciements}

Ces travaux ont été effectués dans le cadre d'un projet de recherches et développement entre le Laboratoire d'analyses et Essais de l'Ecole Supérieure Polytechnique (ESP) de l’Université Cheikh Anta Diop de Dakar et le Laboratoire de Bactériologie et Pathologie Aviaire (LBPA) de l'Institut Sénégalais de Recherches Agricoles (ISRA). Les auteurs tiennent à remercier respectivement les Directeurs de l'ESP et l'ISRA. 


\section{References:}

1. Benajiba M. H., Saoud Y., Lamribah A., Ahrikat M., Amajoud N. \& Ouled-Zian O. (2013). Evaluation de la qualité microbienne des eaux de la nappe phréatique de Martil au Maroc. Revue des Sciences de l'Eau 26 (3): 223-233.

2. ANSD (Agence Nationale de la Statistique et de la Démographie). Situation économique et sociale du Sénégal en 2012. 2015, 11 p.

3. Hounsinou P., Mama D., Dovonou F. \& Alasane A. (2015). Seasonal evolution of the quality microbiological of the natural waters in the township of Abomey-Calavi (South Benin). British Journal of Earth Sciences Research 3(1): 30-41.

4. ISO 19458 (2006). Norme NF EN ISO 19458 (2006) Qualité de l'eau - Échantillonnage pour analyse microbiologique.

5. ISO 4833 -2003. Microbiology of food and animal feeding stuffs Horizontal method for the enumeration of microorganisms - Colonycount technique at $30^{\circ} \mathrm{C}$ (ISO 4833). In: ISO-Microbiology, editor. 3rd ed. p1-9.

6. ISO 5667-3. NF EN ISO 5667-3 (2004). Qualité de l'eau Échantillonnage - Partie 3 : lignes directrices pour la conservation et la manipulation des échantillons d'eau (Indice de classement : T90513).

7. ISO 7027. Norme NF EN ISO 7027 (2007). Qualité de l'eau détermination de la turbidité (Indice de classement : T-90-033).

8. Kouadio L. P., Ekra NB, Atindehou E., Nanou C. \& Monnet D. (1998). Etude de la potabilité des eaux de boisson en sachet vendues aux abords des écoles primaires publiques d'Abidjan. https:/pathexo.societe-mtsi.fr/documents/articles-bull/T91-21766.pdf .

9. NF T90-003 (1984). Essais des eaux - Détermination de la concentration totale en calcium et magnésium - Méthode titrimétrique à l’EDTA.

10. OMS (2017). Organisation mondiale de la Santé, Directives de qualité pour l'eau de boisson : 4éd. intégrant le premier additif; 564 p.

11. Rodier J., Legube B. \& Merlet N. (2009). Analyse de l'eau. 9è Ed, Dunod, Paris $2009 ; 1579$ p.

12. Santsa Nguefack C. V., Ndjouenkeu R. \& Ngassoum M. B. (2018). Pollution de l'eau de consommation humaine et risques sanitaires à court terme : Cas du bassin versant de la Menoua (Ouest-Cameroun). European Scientific Journal 14 (3) : 96-117.

13. Soncy K., Djeri B., Anani K., Eklou-Lawson M., Adjrah Y., Karou D.S., Ameyapoh Y. \& De Souza C. (2015). Évaluation de la qualité 
bactériologique des eaux de puits et de forage à Lomé, Togo. Journal of Applied Biosciences 91: 8464 - 8469.

14. Zerhouni J., Rhazi Filali F. \& Aboulkacem A. (2015). Qualité et facteurs de risque de pollution des eaux souterraines périurbaines de la ville de Sebaa Ayoune (Meknes, Maroc). Larhyss Journal 22 : 91-107. 